\title{
La Armada y el proceso de «profesionalización»
}

\section{Julio Antonio Blázquez García}

Arbor CLXXIII, 682 (Octubre 2002), 239-250 pp.

Tras casi doscientos años de servicio militar obligatorio, las Fuerzas Armadas españolas están afrontando el cambio más profundo de su reciente historia para adaptarse al nuevo modelo de unos Ejércitos totalmente profesionales. Este artículo refleja las diferentes opciones que se presentaban para definir el modelo que se pretendía adoptar, y las medidas que ya se han implantado en las diferentes áreas de la estructura militar para asimilar progresivamente el cambio y la reducción de efectivos, sin merma de las capacidades y eficacia operativa que demandan nuestras necesidades y compromisos en los campos de Seguridad y Defensa. En este sentido, el artículo recoge las líneas generales de actuación que ha emprendido la Armada, potenciando especialmente su Jefatura de Personal en las áreas de captación, retención, motivación y formación de la Marinería y Tropa Profesional, para que los jóvenes sientan identificada su vocación y espiritu de servicio con las peculiares características de la vida en la mar y su plena integración en la Armada.

"Lo importante es que el pueblo advierta que el grado de perfección de su ejército mide con pasmosa exactitud los quilates de la moralidad y vitalidad nacionales».

José Ortega y Gasset

\section{Introducción}

Las Fuerzas Armadas españolas están experimentando una transformación sin precedentes. La incorporación de la mujer, el fin del 
servicio militar obligatorio, la plena profesionalización, la modernización del material, la proyección internacional de Fuerzas y las nuevas estructuras organizativas y de gestión suponen cambios de tal magnitud que están determinando la evolución más profunda, y rápida, en la historia de nuestras Fuerzas Armadas.

Actualmente, el término profesionalización de las Fuerzas Armadas se identifica solamente con el hecho concreto de la suspensión de la prestación del servicio militar obligatorio y la incorporación de un recurso humano de Marinería y Tropa totalmente profesional. Pero el proceso de profesionalización se trata de algo más.

Este proceso comenzó a finales del año 1982 cuando se fijó «el impulso de la profesionalización de los tres Ejércitos» como uno de los objetivos de la Política de Defensa para la II Legislatura (82-86). El fin último era alcanzar una nueva organización militar que permitiera la apertura de canales de confianza entre el Gobierno y las Fuerzas Armadas, vertebrando su dependencia bajo el poder ejecutivo como en cualquier democracia occidental.

Una vez alcanzado este objetivo, se retomó el «impulso de profesionalización» para constituir un proceso completo que conllevase el rediseño de las Fuerzas Armadas españolas, tanto en su aspecto organizativo como en el operativo, para conseguir un avance notable en su integración efectiva en la sociedad española ${ }^{1}$.

Esta es la verdadera génesis del proceso de profesionalización: la integración efectiva de las Fuerzas Armadas en la sociedad. Y el nuevo modelo de Fuerzas Armadas totalmente profesionales, sin personal de reemplazo, es precisamente consecuencia del desarrollo de este proceso.

\section{El motor del proceso}

Las relaciones entre una sociedad y sus Fuerzas Armadas se enmarcan en un conflicto permanente cuya resolución determina las características de la organización militar.

El sistema de una sociedad democrática y de carácter liberal se basa en valores como la libertad y el individualismo, que son significativamente diferentes a aquéllos por los que tradicionalmente se rigen las Fuerzas Armadas. Esto genera una tensión que puede sintetizarse con la siguiente pregunta: ¿Cómo puede una sociedad occidental garantizar su seguridad cuando ello requiere una organización militar cuyos valores no comparte plenamente?.

Las distintas soluciones a este problema se pueden agrupar en tres tendencias, que determinan sus correspondientes modelos de organización militar. 


\section{La Armada y el proceso de «profesionalización»}

La primera solución toma como base que los valores y peculiaridades militares son intocables e inamovibles por constituir la esencia de su eficacia operativa $y$, en consecuencia, de la garantía de seguridad de la sociedad. Esto se materializa en un modelo de organización militar, llamado Institucional ${ }^{2}$, en el que los individuos están plenamente identificados con los intereses de la organización, su compromiso es muy fuerte y tienen un pujante carácter vocacional.

La segunda solución, radicalmente contraria a la primera, aporta la necesidad de una importante evolución de la organización militar para adaptarse a los valores y principios de la sociedad. Los nuevos conflictos, caracterizados por la incertidumbre y los enfrentamientos asimétricos, excluyen la posibilidad de las grandes guerras. Por tanto, esta solución dictamina que los valores militares, diseñados para los enfrentamientos a gran escala, no están justificados actualmente. Por una parte la profesión militar debe desmitificarse y pasar a ser una profesión como las demás y, por otra, la organización militar debe reestructurarse en términos de eficiencia y economía de medios como las demás organizaciones de la sociedad. El modelo de organización militar derivado de esta solución se denomina Ocupacional ${ }^{3}$ y en ella los individuos superponen sus intereses particulares sobre los de la organización, su carácter vocacional es mínimo y su compromiso con la organización, muy débil.

La tercera solución es una vía intermedia entre el estricto criterio de eficacia operativa que persigue el llamado modelo Institucional y el criterio de eficiencia y economía de medios del modelo Ocupacional. Esta solución propone un modelo híbrido ${ }^{4}$ de organización militar que debe mantener unas ciertas peculiaridades ${ }^{5}$ y un esquema organizativo propio que garantice su eficacia y la cohesión de sus miembros; pero también debe saber adaptarse a los principios que imperan en la sociedad, a la que pertenece y sirve, con medidas orgánicas y administrativas similares a otras administraciones públicas. En resumen, este modelo híbrido propone que una organización militar debe poseer los dos componentes, Ocupacional e Institucional, y cada Estado debe saber adecuar la entidad de cada componente según sus características específicas en lo económico, social y político, y sus intereses internacionales $\mathrm{y}$ en materia de seguridad.

La esencia del problema es ¿Dónde fijar el punto de equilibrio entre las dos tendencias? Esta búsqueda del equilibrio adecuado a las exigencias de España es precisamente el verdadero motor del proceso de profesionalización.

Pero si el empuje en una de las direcciones es abrumador, o no existe un cierto contrapeso de equilibrio, la finalización del proceso 
se alcanzará con una excesiva inclinación hacia unos de los extremos, y el correspondiente modelo de organización militar presentará ciertos desequilibrios.

\section{Evolucion de las Fuerzas Armadas Españolas (FAS)}

Hasta el comienzo del «impulso de profesionalización» de 1982, las Fuerzas Armadas españolas se basaban en un modelo orgánico de clara componente Institucional. La Directiva de Defensa Nacional 1/84 marcó el inicio real de la transformación organizativa de las FAS con el objetivo de su integración efectiva en la sociedad, lo que significó una evolución hacia el modelo Ocupacional.

Sin pretender evaluar su bondad, se describen a continuación las sucesivas actuaciones que, a partir de la citada Directiva de Defensa Nacional, han continuado profundizando en el proceso de profesionalización.

- Reforma de la Enseñanza Militar dirigida hacia una mayor integración con el sistema educativo general, con contenidos curriculares que posibilitasen la equivalencia de estudios y titulaciones.

- Diferenciación entre el militar de carrera y el militar de empleo ${ }^{6}$, asignando a éste el carácter de «no permanente».

- Nuevo régimen de los centros docentes militares con disposiciones orientadas hacia objetivos academicistas y para acercar su funcionamiento al de los centros de enseñanza civiles.

- Nuevo sistema de evaluaciones, clasificaciones y ascensos, promoviendo la competitividad entre los individuos, que fricciona con algunos valores formales de la Institución como el compañerismo y la lealtad.

- Nueva política de viviendas militares para impulsar la inserción de los militares en el mercado general de viviendas.

- Adaptación de las retribuciones de los miembros de las FAS al sistema retributivo general del Estado, con incentivos económicos diseñados para las administraciones públicas.

- Reducción paulatina de las compensaciones en especie y servicios, que es un indicador típico de una organización con un cierto componente Institucional.

- Racionalización de estructuras y concentración de unidades, con criterios de economía de medios y eficiencia. 


\section{La Armada y el proceso de «profesionalización»}

- Reducción de las unidades hospitalarias de la red sanitaria militar. En primer lugar se pasó de las 40 existentes a las 14 actuales, como consecuencia de la reducción del despliegue territorial de unidades. En un futuro próximo la red sanitaria militar quedará reducida a 6 unidades hospitalarias ${ }^{7}$.

- Reducción del número de cuadros de mando a un máximo de 48.000 .

- Aumento del número de militares de complemento, de marcado carácter Ocupacional; en el año 2014, al menos el 20 por ciento de los oficiales de las FAS serán de complemento.

- Reducción del número de efectivos de Marinería y Tropa.

Todas estas medidas tienen un objetivo común: la adaptación de la organización militar a los esquemas organizativos de la sociedad y a los principios que en ella imperan: la eficiencia y la economía de medios. Esto, en otros términos, significa un aumento del componente Ocupacional en detrimento del Institucional.

Aunque esta tendencia, con la incorporación de valores y personas de claro componente Ocupacional, puede ser enriquecedora para la organización militar, algunos autores consideran ineludible mantener un cierto componente Institucional que no se debe rebajar para que la eficacia operativa no se vea mermada seriamente ${ }^{8}$.

Quizá debido al devenir histórico de España y a una percepción idílica de inexistencias de riesgos y amenazas externas, la conciencia de Defensa es débil. Tal como indica el senador Muñoz-Alonso ${ }^{9}$ el problema actualmente está en un nivel superior: No puede haber conciencia de Defensa sin una fuerte conciencia Nacional.

Con esta situación, la sociedad española empuja con determinación hacia el sentido Ocupacional de sus Fuerzas Armadas. La profesionalización es popular sólo por lo que tiene de aligeramiento de cargas de recursos financieros y humanos, no por lo que supone de mejora en el rendimiento ${ }^{10}$. Esta es la fuerza predominante en la búsqueda del adecuado modelo híbrido de organización militar.

Poco a poco se avanza hacia una homologación de la organización militar con los demás sistemas organizativos de la sociedad ${ }^{11}$. Esta tendencia Ocupacional, que está acorde con la evolución de las Fuerzas Armadas de la mayoría de los países de nuestro entorno, implica una significativa reducción del componente vocacional y altruista de la pertenencia a las FAS en aras del pragmatismo y el afán remunerativo.

Hasta aquí se ha expuesto la evolución general, incertidumbres y retos que presenta de forma global el proceso. Pero dentro de éste, la suspensión de la prestación del servicio militar obligatorio, y la incor- 
poración de los nuevos marineros y soldados totalmente profesionales, constituyen los hitos más destacados en la actualidad, que comúnmente se engloban con la expresión «plena profesionalización». Con este nuevo modelo se sustituye el sistema de conscripción por un reclutamiento voluntario.

\section{La plena profesionalización y el reclutamiento voluntario}

El 31 de diciembre de 2001 se cerró una etapa histórica de 200 años de servicio militar obligatorio, que la Constitución de Cádiz había establecido en 1808. En 1998 la Comisión Mixta Congreso-Senado para la plena profesionalización dictaminó la creación de unas Fuerzas Armadas totalmente profesionales con una entidad numérica delimitada por la horquilla 102.000 - 120.000 efectivos de Marinería y Tropa. El primer objetivo se concretó en 110.000 efectivos, y posteriormente se situó en el extremo más bajo de la citada horquilla legal, 102.000.

La justificación política de esta importante decisión, la «total profesionalización» de las FAS, se basó en dos argumentos principales. El primero fue de carácter social: la sociedad española, especialmente la juventud, empezaba a dar serias muestras de un falta de comprensión y una disposición desfavorable hacia el servicio militar obligatorio, que se reflejaba en los crecientes índices de la objeción de conciencia. Y existió también una razón estratégica: la anterior concepción de la Defensa, que estaba enfocada exclusivamente a la protección del territorio nacional, permitía circunscribir el empleo de los soldados de reemplazo a nuestra geografía; pero la nueva idea de la Defensa, enmarcada en los principios de seguridad compartida y defensa colectiva del ámbito occidental, y como parte de la Acción Exterior del Estado, implicaba una elevada proyección de Fuerzas y misiones en teatros muy alejados de nuestras fronteras que la sociedad no aceptaba asumir con personal de reemplazo.

El nuevo sistema de reclutamiento voluntario abarca tres actividades esenciales para la obtención del recurso de Marinería y Tropa: la captación de jóvenes españoles, la retención o permanencia y su reincorporación a la vida civil. Estos son los tres eslabones del reclutamiento, de manera que la bondad del sistema puede medirse por la «resistencia» del eslabón más débil.

Las FAS disponen actualmente de un total de unos 80.000 efectivos de Marinería y Tropa, apreciándose últimamente una cierta desaceleración en el sistema de reclutamiento, lo que tendrá como consecuencia que el objetivo final de 102.000 efectivos se alcanzará dos o tres años 
más tarde de la fecha prevista . Las principales razones de esta ralentización son:

- La reducción de la «bolsa de aspirantes» dado el fuerte flujo de incorporaciones durante los últimos años.

- La próspera situación económica española que tiene un significativo impacto en una oferta de trabajo tan importante como la de las Fuerzas Armadas.

- Una situación demográfica desfavorable, con una sustancial y progresiva disminución del segmento de población entre los 18 y 27 años.

- La reticencia de los jóvenes españoles a la movilidad geográfica, que limita las fuentes de aspirantes a las localidades donde están ubicadas unidades militares.

Con la citada tendencia Ocupacional, marcada por la sociedad y acorde con la situación de los países de nuestro entorno, uno de los principales aspectos de la captación es el nivel retributivo. Aunque el sueldo percibido inicialmente no está por debajo de lo que ofrece el mercado laboral a trabajadores sin una alta especialización formativa, si éste se asocia con el duro régimen de vida que conlleva la profesión militar, la competitividad de la oferta de empleo de las Fuerzas Armadas se reduce significativamente.

Esto tiene una manifiesta influencia en la retención o permanencia. Algunos jóvenes que se incorporan con actitudes meramente ocupacionales, y pragmáticas, perciben que sus intereses personales chocan con las exigencias de disponibilidad, sacrificio y abnegación inherentes a la vida militar. Esto les lleva a finalizar su compromiso militar antes del deseable, y mínimamente rentable, período de permanencia.

Para incidir en la mejora de este binomio «nivel retributivo-régimen de vida» se ha aprobado recientemente un nuevo reglamento de retribuciones cuya finalidad es fomentar la renovación de compromisos de los marineros y soldados. También se están llevando a cabo importantes inversiones en infraestructuras y en la externalización de tareas no propiamente militares, orientadas a mejorar la calidad de vida de nuestros marineros y soldados.

Por otra parte, el Ministerio de Defensa va a determinar un sustancial porcentaje de marineros y soldados que podrán adquirir el carácter permanente en las FAS, lo que proporcionará una mejora en la visión de futuro estable, y que redundará en beneficio tanto de la captación como de la retención. 
Una vez alcanzada la estabilidad numérica de efectivos, las necesidades anuales de reclutamiento se cifran en 12.000 jóvenes y, naturalmente, existirá un flujo similar de salidas que se distribuirá entre los que accedan a las academias de suboficiales, los que ingresen en la Guardia Civil y los que deberán reinsertarse en el mundo laboral civil.

La faceta de «reincorporación a la vida civil» se enfoca proporcionando una formación educativa adecuada, como valor añadido a la formación y experiencia militar. Existe ya una homologación de las titulaciones obtenidas en la Enseñanza Militar con el nivel de Formación Profesional del sistema general de educación.

La apertura de convenios con grupos empresariales y la valoración de la experiencia y servicio en las FAS para los diferentes baremos de ingreso en la Administración Pública, que actualmente se está tratando con el Ministerio de Administraciones Públicas, facilitan también la reincorporación laboral a aquéllos que finalizan su compromiso con las Fuerzas Armadas.

A lo largo de esta exposición se ha podido observar cómo la Política de Personal es el elemento director del proceso de profesionalización. Debido a las condiciones de ajuste presupuestario y a la necesidad de racionalización de medios, la planificación de medidas y el control de objetivos de la Política de Personal, incluido el Reclutamiento, se realiza desde los Organos Superiores del Ministerio de Defensa con la estrecha colaboración de los Cuarteles Generales que, posteriormente, son los responsables de desarrollar, ejecutar y gestionar todas estas medidas en sus específicos ámbitos de actuación.

\section{El impacto de la «profesionalización» en la Armada}

La Armada, aunque unida de forma indisoluble a este proceso, tiene también un margen de maniobra para materializar y adaptar las medidas generales a las peculiaridades propias de la vida naval, determinadas por su medio de trabajo operativo: la mar y los buques.

El proceso de profesionalización ha sido enfocado en la Armada con un profundo replanteamiento de su Organización. Los principios organizativos que tutelaban la Armada se basaban en teorías orgánicas de los años sesenta, concretamente las de Fayol y Weber, y sus enfoques se reducían a la «estructura» y las «tareas» para afrontar la necesaria división del trabajo. La adaptación de la Armada a las modernas teorías de la organización impuso cinco nuevos enfoques: la Estructura, los Procesos de Trabajo, las Personas, el Entorno y la Tecnología. Se per- 


\section{La Armada y el proceso de «profesionalización»}

sigue una organización moderna, a caballo entre la tradición y la innovación, que pueda asumir con flexibilidad la única certeza de la situación actual: la incertidumbre y el cambio constante.

Uno de los principales objetivos que persiguen estos cambios organizativos es el capital humano de la Armada. La Armada ha asumido plenamente que las personas son el elemento más potente de su Organización y, de esta manera, la Jefatura de Personal ha dejado de ser un mero órgano gestor para constituirse en un departamento de recursos humanos de carácter estratégico que afronte tareas tan novedosas como la Motivación de sus miembros, dentro de un innovador proceso de trabajo: la integración de las personas de la Armada.

La vertiginosa implantación del nuevo modelo del militar profesional de Marinería y Tropa impuso en la Armada el nuevo sistema del reclutamiento voluntario y sus tres áreas: captación, retención y reincorporación a la vida civil.

La Armada modificó con prontitud sus estructuras para hacer frente a este importante reto. Se creó una Dirección con dedicación exclusiva a esta actividad y se potenció una estructura periférica que ejecutase las líneas directivas en cada una de las citadas áreas. La Armada ha maximizado sus esfuerzos humanos y financieros para que, dentro de los planteamientos y condiciones marcadas por la Política de Defensa, se alcancen los objetivos numéricos de Marinería y Tropa.

El Almirante Jefe del Estado Mayor de la Armada definió, en el documento "Líneas generales de la Armada» ${ }^{12}$, los aspectos fundamentales que deben regir el Reclutamiento para presentar una oferta de trabajo atractiva y rigurosa, a saber: perspectivas de futuro, integración efectiva en la Armada, satisfacción en el trabajo y calidad de vida. Estos aspectos constituyen las primeras prioridades en la asignación de recursos específicos de la Armada.

En esta línea cabe destacar medidas tan llamativas para nuestra cultura organizativa como la racionalización de los regímenes de guardias en las unidades, la compensación por navegaciones y maniobras durante jornadas no laborables, programas de atención a la familia, construcción de guarderías, residencias y espacios de ocio para nuestros marineros y soldados, impulso del Liderazgo en las relaciones de mando y jerarquía, y la orientación profesional y tutorías personalizadas para los miembros de la Armada.

Se están realizando campañas informativas internas para incidir en la «satisfacción individual en el trabajo» de nuestros marineros y soldados mediante una adecuada concienciación de los oficiales y suboficiales de la Armada. Se trata de implantar la idea de que los 
nuevos marineros no constituyen una variación retribuida del servicio militar obligatorio, sino que son profesionales como los demás miembros de la Armada. Es necesario asignarles tareas acordes con su formación que constituyan retos factibles, con un cierto grado de responsabilidad e iniciativa, de manera que perciban que su trabajo es importante para la Armada.

La Armada se ha propuesto también que la formación y la experiencia en trabajos técnicos sean importantes atractivos de captación y retención. Nuestros buques, submarinos, aviones, helicópteros, unidades de Infantería de Marina, y las correspondientes infraestructuras de apoyo, poseen sistemas y equipos con las tecnologías más avanzadas que permiten un aprendizaje y experiencia técnica muy valoradas en el sector civil.

En el campo de la formación, la Armada está implantando un nuevo proyecto de modernización de la Enseñanza Naval para que, basándose en las nuevas tecnologías de información y comunicaciones, se adapte el entorno espacio-tiempo de la docencia, anclada hasta ahora en un exclusivo carácter presencial, a la vida naval de nuestros marineros y soldados que se caracteriza por el constante devenir.

Sin embargo, y a pesar del enorme esfuerzo realizado para hacer frente al nuevo reto del reclutamiento voluntario, la Armada ha encontrado mayores dificultades que los otros dos Ejércitos para la captación y retención de sus efectivos de Marinería y Tropa ${ }^{13}$. ¿Cuáles son las razones? Sin lugar a dudas las propias características de la vida naval y de la Armada que, a pesar de las gratificantes experiencias personales y profesionales que proporciona, la distinguen por su exigencia de los otros componentes de las FAS. En este sentido cabe destacar la dureza del régimen de vida a bordo de los buques, la separación del entorno familiar durante largos períodos de operaciones y maniobras en teatros alejados, y una elevada movilidad geográfica durante la trayectoria profesional.

Esta situación genera un aspecto negativo añadido que se manifiesta con el "círculo vicioso» siguiente: menos personal, mayor carga de trabajo, menos personal. Por otra parte, el lento ritmo de incorporaciones también presenta un lado positivo, ya que facilita unas trayectorias profesionales más equilibradas y de una mejor progresión.

La Armada cuenta actualmente con 14.000 efectivos de Marinería y Tropa, es por tanto necesario realizar un último esfuerzo para conseguir un crecimiento que permita alcanzar el objetivo legal de los 18.813 efectivos. Dada la actual ralentización del crecimiento, a pesar de la polarización de los empeños de la Armada para la culminación 


\section{La Armada y el proceso de «profesionalización»}

con éxito de este proceso, parece conveniente la inserción de nuevos factores que produzcan un revulsivo en el Reclutamiento de la Armada.

Por una parte, se requieren mayores recursos financieros que permitan consolidar las acciones ya iniciadas para incidir en la calidad de vida de nuestros marineros y soldados y hacerla acorde con las condiciones medias de la sociedad, como son la externalización de tareas no militares, nuevas infraestructuras, y una formación con «valor añadido", tanto para la promoción interna como para la reinserción en la vida civil.

$\mathrm{Y}$, por otra parte, parece necesario disminuir la tendencia Ocupacional que está marcando el carácter pragmático de la oferta de empleo de la Armada, y de las Fuerzas Armadas en general, y apelar a la vocación y al espíritu de servicio de los jóvenes españoles. En este sentido, y dado el especial régimen de vida que ofrece la profesión naval, cabe también replantearse su oferta laboral, que hasta ahora ha estado orientada como un puesto de trabajo más dentro del mercado laboral y que ha generado expectativas confusas.

La pertenencia a la Armada conlleva un modo de vida especial, muy exigente, pero a la vez muy gratificante y que no puede ser homologado a un trabajo más de la sociedad. Es necesario concentrar el esfuerzo en una política dirigida a mejorar los aspectos de integración, satisfacción en el trabajo, perspectivas de futuro, formación y calidad de vida, para presentar a los jóvenes una oferta atractiva y rigurosa en la que encuentren satisfechas sus aspiraciones con el orgullo de pertenecer a la Armada.

\section{Notas}

1 Ministerio de Defensa. Memoria de la II Legislatura (1982-86), Ministerio de Defensa, 1986, p. 17.

2 Huntington, Samuel P., The soldier and the state. Cabridge, Massachusetts. Belkap Press. 1985.

3 JANOwitz, MoRRís, El soldado profesional, Madrid, Ministerio de Defensa, 1990.

4 Moskos, Charles, Lo militar ¿Más que una profesión?, Madrid, Ministerio de Defensa, 1991.

5 Quizá la peculiaridad más llamativa se refleje en el artículo 28 de la Constitución Española.

6 Actualmente denominados militares de complemento, a los de categoría de oficial, y militares profesionales de Tropa y Marinería.

7 Comparecencia en el Congreso del Sr. Ministro de Defensa para informar sobre el proceso de profesionalización. Diario de sesiones $n^{\circ} 82 / 2000$. 19 octubre de 2000 . p 2232. 
8 MOSKOS, op cit. p. 31 .

9 Muñoz-Alonso, Alejandro. "Renovación a fondo de la política de Defensa». Revista española de Defensa. Núm. 144, Febrero de 2000. P. 40.

Alejandro MuÑoz-Alonso fue presidente de la Comisión de Defensa del Congreso de los Diputados durante la VI Legislatura.

10 COMA, MANUEL. «Seguridad y Defensa, una disciplina académica aún incipiente». Revista española de Defensa. Núm. 144, Febrero de 2000. P. 53.

MANUEL COMA es profesor de la UNED y presidente del Grupo de estudios estratégicos.

11 GiL MuÑoz, Carlos, Apéndice. Lo militar ¿Más que una profesión?, Madrid, Ministerio de Defensa, 1991. P. 412.

12 El documento "Líneas Generales de la Armada" establece la política de la Armada en todos sus campos de actuación con un horizonte del medio plazo, marca las prioridades para la asignación de recursos y permite a las autoridades subordinadas contar con criterios para la toma de decisiones que orienten los esfuerzos de la Armada hacia objetivos únicos.

13 Comparecencia del Ministro de Defensa, Op cit, p. 2230. «...la escasa cantidad de solicitudes en los destinos de la Armada y la Legión, frente al excesivo número de solicitudes para el Ejército del Aire y la Guardia Real....". 